\title{
Teleservice - Opening Doors to the World Market
}

\author{
Dr. -Ing. Dipl.-Wirtsch.-Ing. S. Stender, \\ Fraunhofer Institute for Manufacturing Engineering and \\ Automation (IPA), Nobel Str. 12, 70569 Stuttgart, Germany \\ Tel. $+49(0) 711-9701973$ \\ Fax. $+49(0) 711-9701002$ \\ e-mail sts@ipa.fhg.de
}

\begin{abstract}
In today's turbulent markets it is important that machine manufacturers are able to react quickly and effectively to changing circumstances. One potential area of improvement is that of service in maintenance. Instead of merely offering services in the classical areas of maintenance like inspection and repairs, manufacturers should listen to the specific needs of their clients and also provide guidance in finding the optimum structure for the client's production processes. The Service Support System, developed by the Fraunhofer IPA, provides a joint service platform between machine manufacturers and operators and is the ideal tool to help machine manufacturers expand their after-sales services, enabling them to meet their client's need more effectively, while strengthening their position amongst competitors.
\end{abstract}

Keywords

Teleservice, production management, maintenance, service, operations 


\section{WHY TELESERVICE?}

Increasing competition in the world market has given greater significance to the technical services offered by machine and plant manufacturers. In fact, they are expected to react more quickly and efficiently to their clients service requirements as the breakdown of highly automated machine systems can lead to large losses in invested capital and manufacturing capacity. However, hardly any modern machine manufacturer today can promise every client world wide an immediate response to their service needs and manufacturers are dependent on their own maintenance department. In addition, the ensuing costs and the time lost by sending a maintenance technician to the client can sometimes be out of proportion to the actual problem faced by the client.

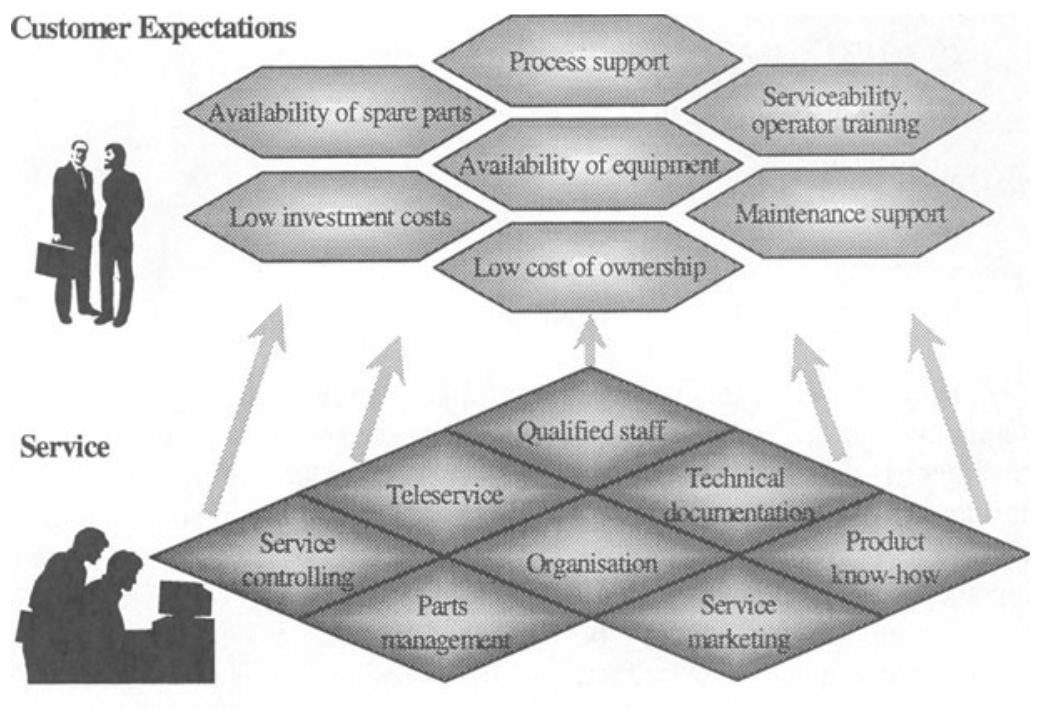

Source: Institute for Manufacturing Engineering and Automation (IPA)

Figure 1. Building Blocks for Customer-Oriented Service

Many machine and plant manufacturers see the solution to these challenges in the use of Teleservice applications. Teleservice is a long distance linking up of the service provider with the client's machine controls. It also includes the application of telecommunication services for maintenance and related functions like machine installation and adjustment. If such applications are used in manufacturing functions, one speaks of Telemanufacturing. 


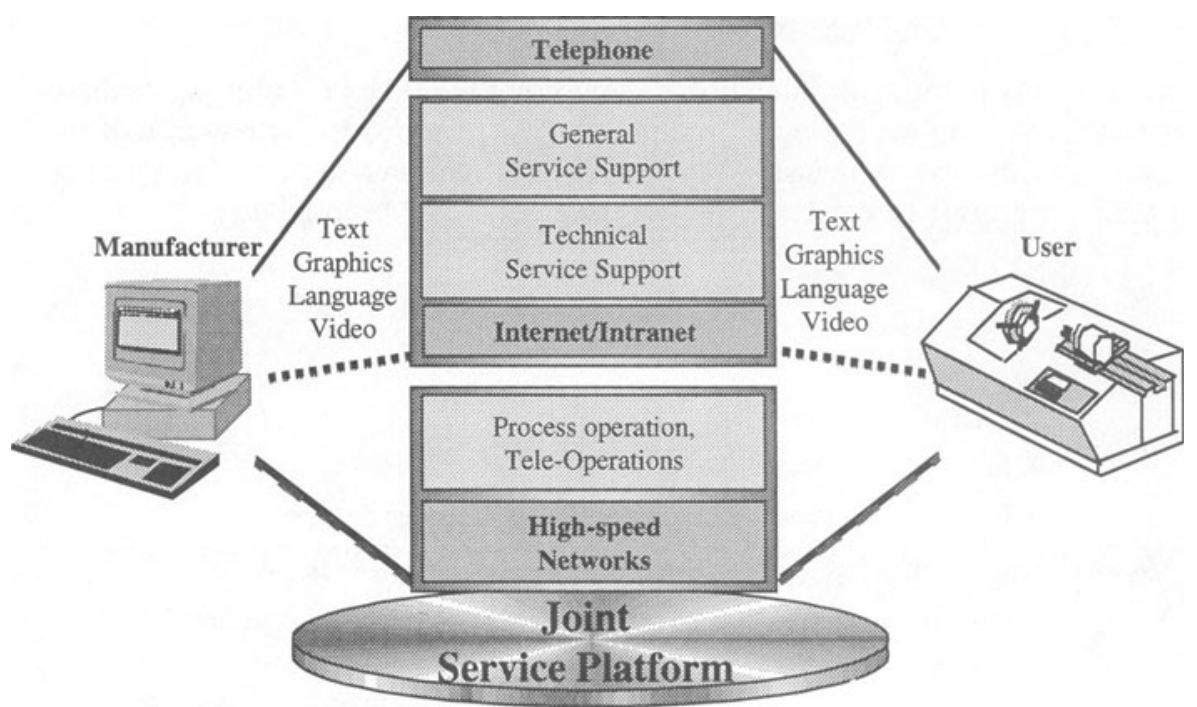

Source: Institute for Manufacturing Engineering and Automation (IPA)

Figure 2. Service Support through Media and Networks

\section{HOW IT ALL BEGAN}

The use of Teleservice in a rudimentary form began twenty years ago when machine builders would communicate instructions to the operators via the telephone. This was only possible when the machines running were not too loud. The basic concept behind Teleservice today has remained more or less the same with the use of analogue connections and a modem. However, more flexibility has been introduced through the use of programmable storage controlling systems (SPS) which are more modular in their set up. Through their combination with machine and field buses, fractal machine control has also become possible. Similar to the nervous system, controlling tasks can be distributed from a central computer to decentralised components right down to the actor/sensor level. The development of computer-based controlling has made it possible to put this concept into action. 


\section{DIFFICULTIES IN APPLICATION}

One difficulty in the application of Teleservice has been the lack of a standardised system for gaining access to the machine controls, but due to the pressure of the market and the use of industrial computers with real-time systems for machine control, the process of standardisation has been accelerated enormously.

\section{- On-site infrastructure often lacking \\ - Investment and maintenance costs partially overestimated \\ w Inadequate telecommunication infrastructure in foreign countries}

virtual machines: linkage of production plant with teleconununication networks
Machine manufacturers have specific control units; interfaces are required
Uncertainty of user concerning process data and security
Disappointing experiences the user has had with the manufacturer's Telesevice,
concerning the cost/benefit ratio
the possibly necessary sensor system increases the cost of the machine
virtual experts: On-site information technology support through teleconmunication
- Standardisation of material elements (Data formats, numerical systems, functional
characteristics, connections with lists of production sites)
- Frequently lacking digital technology in technical documentation

Source: Institute for Manufacturing Engineering and Automation (IPA)

Figure 3. Existing Obstacles to Greater Application of Teleservice

\section{THE VISUAL ADVANTAGE}

"Transparent" machine controlling is the latest in modern technology although few machine manufacturers make use of it to offer their clients services such as machine installation, maintenance and assistance in operation. With the help of software applications like pcAnywhere or Reachout it is possible for the service technician to view the screen of the computer controls of the machine on his Teleservice station and then gain access to the machine under inspection. On the client's side, the operator is able to follow all the adjustments carried out by the service technician. For machine and process diagnosis, it is possible to transfer sensor data for analysis and evaluation by the experts at the machine manufacturers. 
This transfer of visual images (still or moving) is invaluable for the long-distance diagnosis of complicated machine disruptions. Especially when talking about Teleservice on a global scale, the power of visual images to help overcome language barriers should not be underestimated.

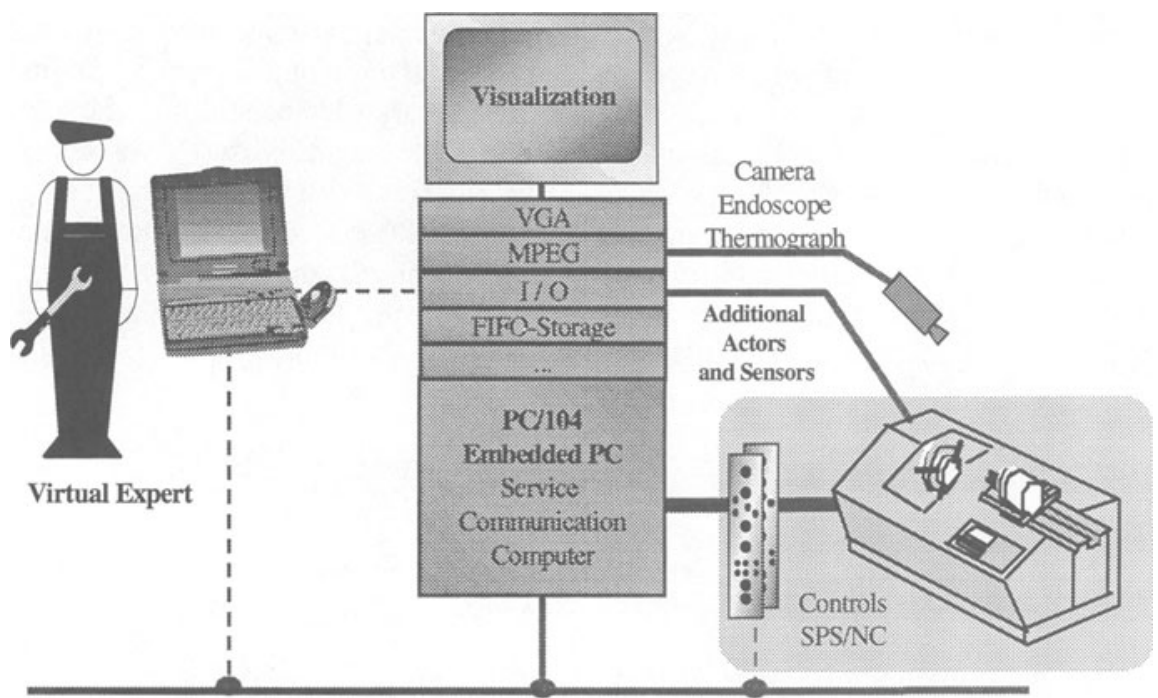

Field bus - LAN - WAN - Internet - ISDN Network

Source: Institute for Manufacturing Engineering and Automation (IPA)

Figure 4. Process Visualization using Video and Sensor Data

In many cases, stills made with modern digital cameras are often sufficient. The images can then be transferred by File Transfer via modem or through the Internet protocol TCP/IP. The latter, which works with satellite technology has the advantage that it does not depend on the availability of a telephone line and image transfer is also possible with a laptop or a mobile phone.

Moving images make it possible to analyse processes within the production line which go beyond a simple machine diagnosis. It is essential that the images be transferred in real time so that both the machine operator and the service technician can follow the whole situation as it happens. For the transfer of sharp and quality images, high image frequency and ISDN cabling, which may not be available in certain parts of the world, is strongly recommended. 


\section{THE IPA PROJECT}

\subsection{The S3-Ba-Wü Project}

One of the current Teleservice projects at the Fraunhofer Institute for Manufacturing Engineering and Automation (IPA) is the "S3-Ba-Wü" This project, sponsored by the State of Baden Württemberg and Deutsche Telekom, is being carried out in cooperation with several regional companies. The aim is to develop a prototype client-oriented solution for service problems through Teleservice. Focus is being given to the conception of a system for the transfer of still and moving images. Various services (Spare part identification, diagnosis, etc.) are being evaluated for suitability as Teleservices while different systems are also being tested for these services. Apart from the costs, the available image frequency is one of the most important criteria for testing each system in varying service scenarios. It is evident that compromises are necessary when trying to find optimum image frequency, frequency range and image resolution at an affordable price.

\section{Customer-Orientation through organisational and technical flexibility}

- Cost-effective global presence

- Accessing and storage of technology information independently of time or place

- Immediate access to information independent of size or origin

\section{Greater quality of service through multimedia technology}

- Rapid information feedback / over large distances through multimedia technology

- Rapid diagnosis of complex machines through qualified support

\section{Connection with customer using appropriate information technology}

- Quick and easy processing and administration of client data based on standards

- Standard-based adaptation to company-specific requirements for optimum system structuring

Source: Institute for Manufacturing Engineering and Automation (IPA)

Figure 5. Objectives of the S3-BaWü Project

\subsection{The Service Support System}

On the basis of these studies, IPA has developed the Service Support System, which provides a joint service platform between machine manufacturers and 
operators. It consists of three main components which work together as a complementary, supportive network:

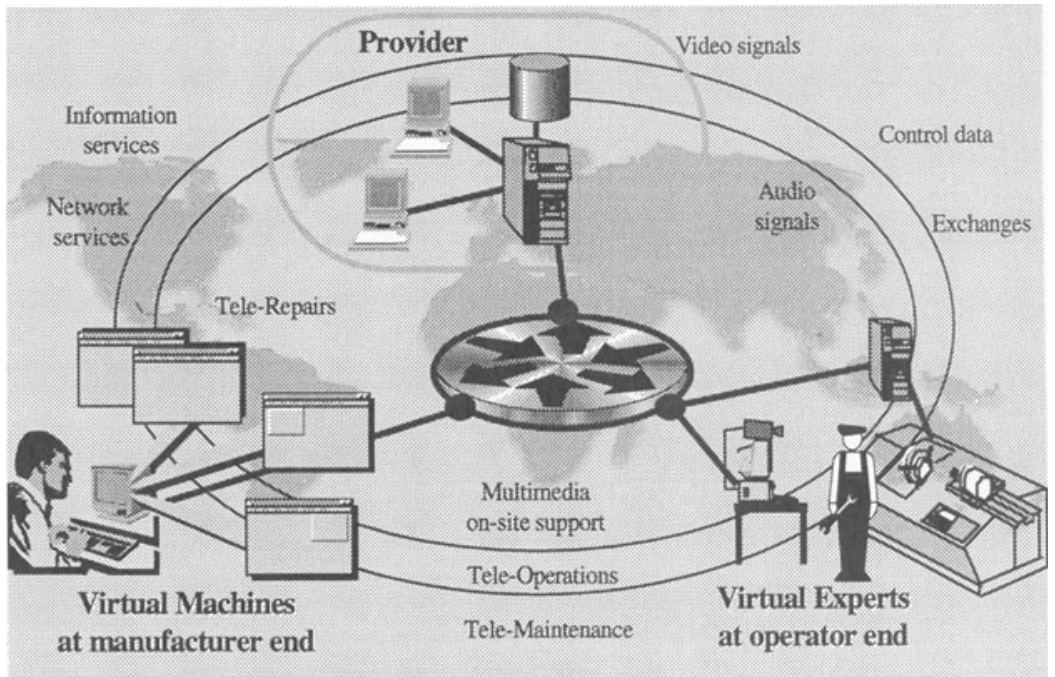

Source: Institute for Manufacturing Engineering and Automation (IPA)

Figure 6: The service Support System

\section{Virtual machines at the manufacturer end}

All the relevant information and tool for the diagnosis and elimination of machine and process disruptions are located at the manufacturers end. In addition to the actual management of disruption, using the online transmission of sensory and visual information, the service technician can monitor and control production processes in accordance with the requirements set by the client. This so called teleoperation is supported by the latest developments in the field of automation and bus technologies. 


\section{Virtual experts at the operator end}

Information specific to the machine or the manufacturer is made available to the operator on site. This makes it easier for the manufacturer's service workers to identify and eliminate disruptions in the machine process when they are called in by the client. The electronic linkage with an online database run by the manufacturer provides the operator with further informational support.

\section{On-Site Information Technology Support through Telecommunication}

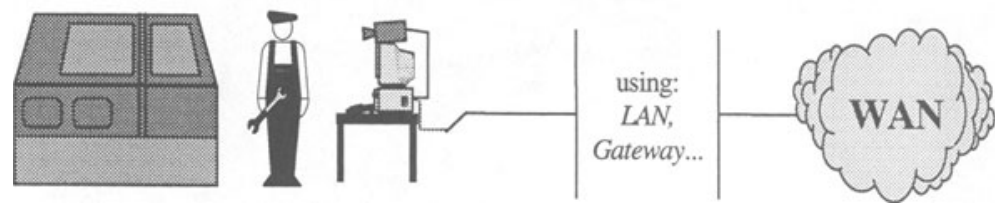

Applications: - Access to technical documentation (system configuration, hydraulic circuit and wiring scheme, diagrams, diagnosis checklist, maintenance instruction manuals (Moving picture), ...)

- Material management (Spare parts identification and ordering)

- Conference of experts for diagnosis (Video conferencing)

- Access to central expert systems (e.g.. case-specific diagnosis systems)

- Contract management

- ...

Source: Institute for Manufacturing Engineering and Automation (IPA)

Figure 7. The Role of the Virtual Experts

\section{The virtual service network}

This aims to improve communication between machine manufacturers and operators through the use of multimedia information technology. It is made up of a technical component which relies on the use of Internet technology and an organisational component which provides an essential infrastructure for small manufacturers. The organisational component also offers a joint software platform which includes programs such as EDM, CAD and MRP. Additional support is also given in various aspects of administration, information, security and statutory rights. 
The benefits of the Service Support System include reduced service expenses through the avoidance of unnecessary visits by service/maintenance workers. At the same time, the services provided are more client-oriented and more flexible. Even less qualified service staff can become virtual experts, because all the information required to rectify a disruption is easily accessible in an expandable online database. This knowledge database also facilitates the effective identification of spare parts so that false deliveries can be avoided.

\section{THE WAY AHEAD}

In order for Teleservice to work, it is not enough to just have the right technology. Teleservice should to be integrated within a machine manufacturer's service range and also within the client's organisation. Questions such as whether Teleservice should be introduced to cut maintenance costs or if it should be offered as an extra service for the client are to be examined from the start. It is also important to take the administration, personnel and running costs into consideration; if the service is to be offered world-wide, it is necessary to have specialists on hand around the clock. These extra expenses may be balanced out by the savings made by not having to send service technicians to the client. With the right strategy, both the machine manufacturer and buyer or operator can benefit from Teleservice.

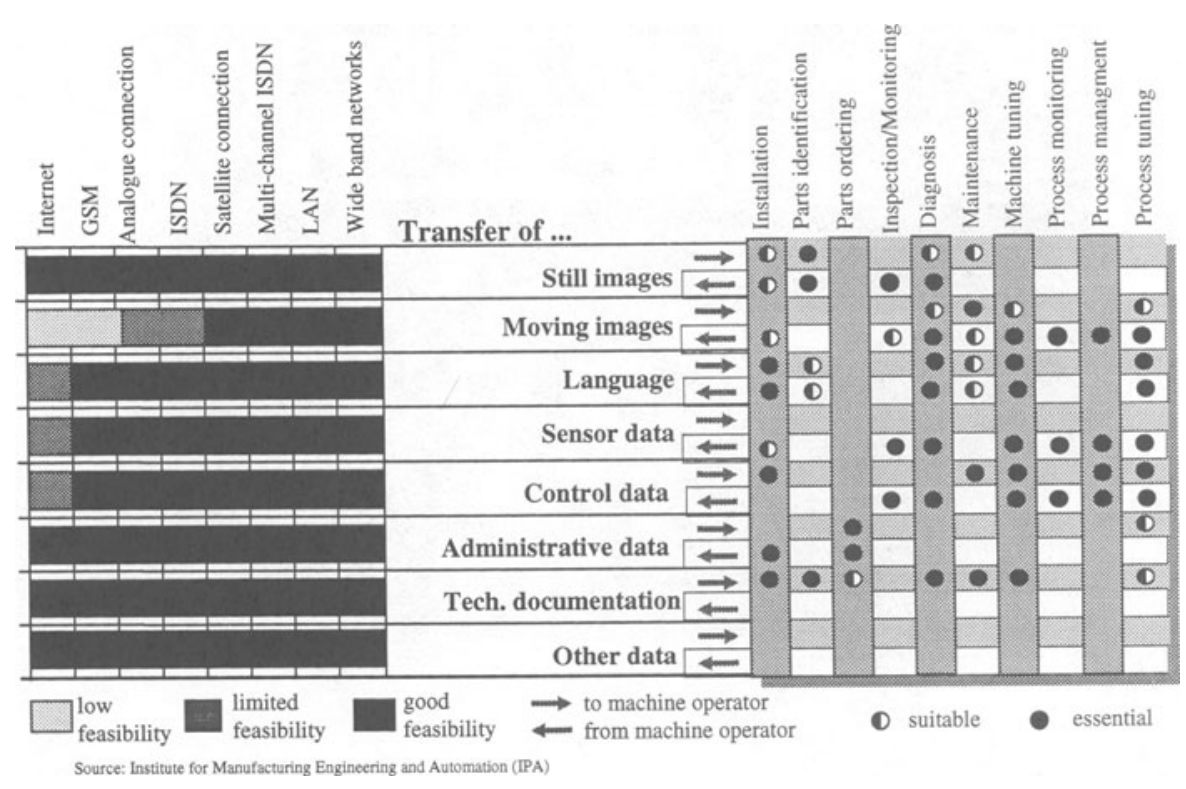

Figure 8. The Flow of Information During Teleservice 
Once the appropriate infrastructure has been set up, the potential benefits of Teleservice are enormous. The machine manufacturer is able to support the client in many ways including the provision of up-to-date technical information about the product and by allowing access to technical documentation and instructions to maintenance problems for reference or training purposes. For example, if technical documentation support is required for the identification of spare parts, then it should be possible to access the parts ordering department of the manufacturer by mouse click. In addition, information concerning technical disruptions, quality control etc. can be passed from the operator back to the manufacturer for analysis.

The application of Teleservice functions for the control of production processes gives rise to more difficult challenges. Such Telemanufacturing applications are most suitable for decentralised, spatially detached production areas with stabile, controlled processes, especially during low labour-force shifts. This technology has been in use for some time in the power industry where process changes are relatively slow.

A live presentation of how Teleservice can work was made during the Tools Fair (EMO) in September 1997. In this example, a dry grinding process at the Institute for Machine Research in Braunschweig, Germany was steered from the IPA stand via Internet. The process temperature could be monitored through the transfer of thermographs and it was also possible to regulate the most important process parameters.

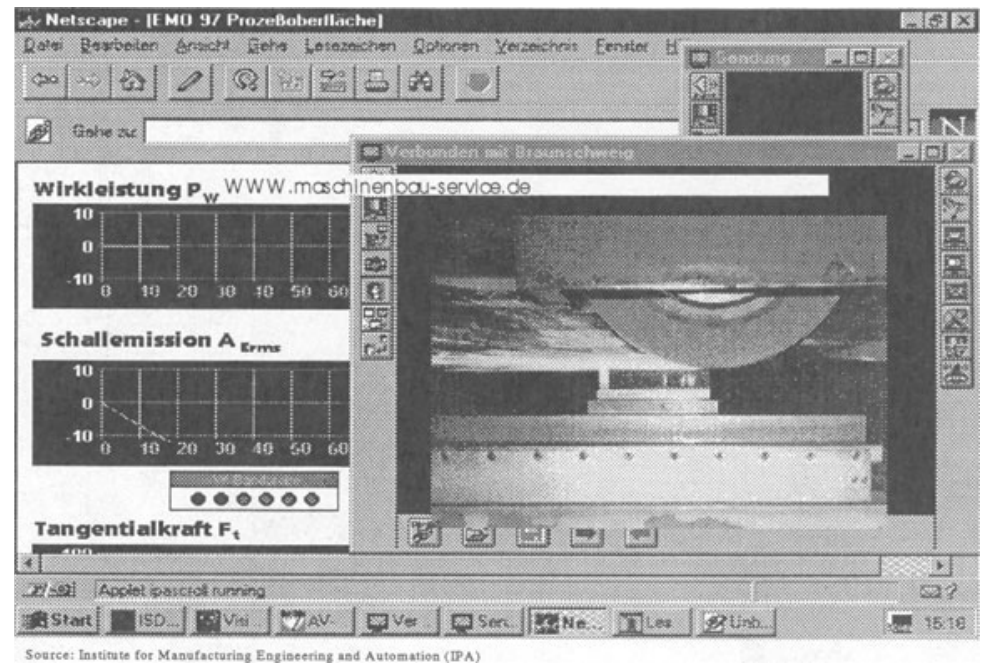

Figure 9. The Use of Thermography for Process Management 
For both Teleservice and Telemanufacturing the area of responsibility covered by each co-operator, especially when in different companies, is a very important point to consider. It is necessary to formally clarify what services the client expects and what services should be carried out by the manufacturer within which time limit. The contract should also specify who is to bear the costs if it is not possible to repair the breakdown quickly or if subsequent problems occur due to deficient teleservicing.

\section{CONCLUSION}

The application of Teleservice provides many benefits to the manufacturing industry. A company can increase its presence on the global market without having to invest in manned service stations to deal with the problems of clients in different areas of the world. Teleservice enables even small and medium sized machine manufacturers respond to clients needs in a quicker, more specific and more effective way. With the application of simple tools it is already possible to provide a great increase in customer benefits. A lot of research is in progress and there are still areas requiring further development before sophisticated, industrially viable solutions can be put into practice. However, the increasing significance of the service sector and the opportunities provided by modern information technology makes it impossible to ignore the potential of Teleservice.

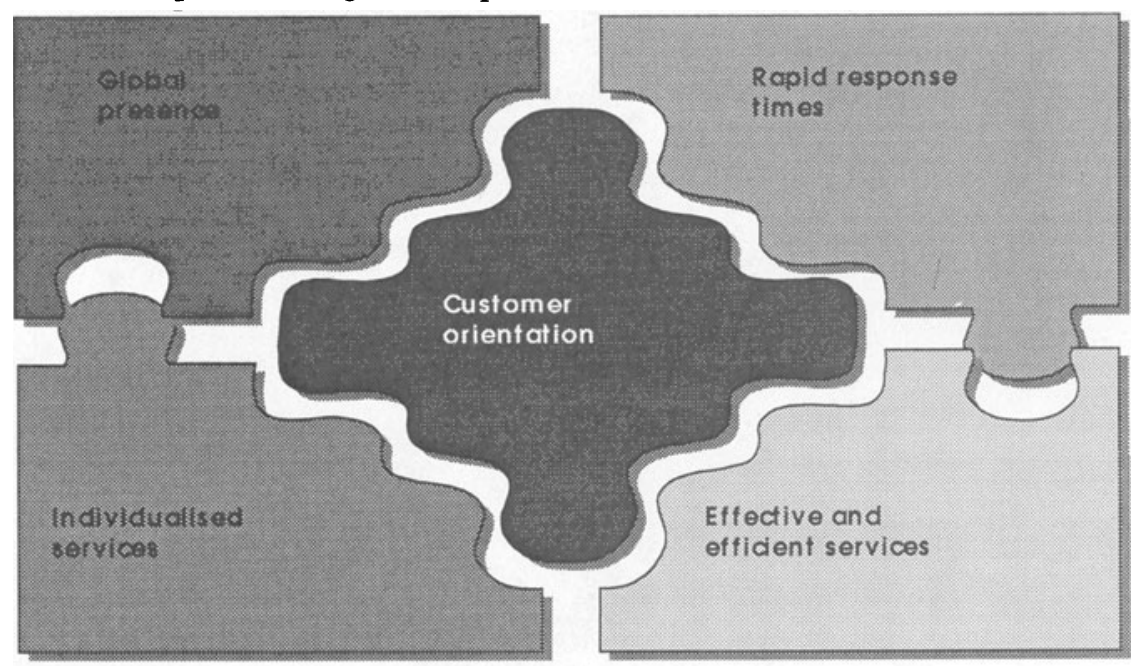

Source: lastitute for Manufacturiag Eagineeriag and Automation (IPA)

Figure 10. The Benefits of Teleservice 


\section{BIOGRAPHY}

Dr.-Ing, Dipl.-Wirtsch.-Ing Siegfried Stender is Head of the Department of Production Management at the Fraunhofer Institute for Manufacturing Engineering and Automation (IPA) in Stuttgart, Germany where he has been actively involved in industrial projects for over ten years. His areas of expertise include maintenance management, production organisation, fractal company structures and logistics. Dr. Stender has led many projects involving these topics in conjunction with leading companies from a variety of branches such as electric power supply, automobile and aviation, electronics, hospitals, public transport, engineering and food processing 